\title{
Identification of Circulating Tumor Cells and Chemokine Receptor CCR6 as Noninvasive Biomarkers in Egyptian Patients with Hepatocellular Carcinoma
}

Mohammed F. Elshal ${ }^{1}$, Manal O. Elhamshary ${ }^{1}$, Morad M. Mahmoud ${ }^{1}$ and Neama M. Lotfy ${ }^{2}$

${ }^{1}$ Molecular Biology Department, Genetic Engineering and Biotechnology Institute, University of Sadat City,

Sadat City, Egypt, ${ }^{2}$ Clinical Pathology Department, Faculty of Medicine, Ain Shams University, Cairo, Egypt

*Corresponding Author: Neama Lotfy, Mobile: (+2) 01008334082, E-mail: neama_lotfy@yahoo.com

\section{ABSTRACT}

Background: Circulating tumor cells (CTCs) are cancer cells in the circulation that are derived from the original tumor or metastatic foci. Chemokines and their receptors have major roles in hepatocellular carcinoma (HCC) metastasis. They also represent a very promising group of markers to detect CTCs in HCC patients. The CCL20CCR6 axis promotes cancer proliferation, migration, and tumor remodeling through immune cell control.

Objective: This study aimed to identify the role of CTC and CCR6 in hepatocellular carcinoma patients.

Patients and methods: The study included 91 subjects; 71 HCC patients and 20 normal individuals. Routine laboratory investigations included CBC, PT and INR, ALT, AST, bilirubin, albumin, alpha fetoprotein, and creatinine in addition to detection of chemokine receptor 6 (CCR6) RNA by real time PCR and CTCs by flow cytometry as $\mathrm{CD} 45^{-} \mathrm{CK} 19^{+}$cells were performed for all subjects enrolled in this study.

Results: There was significant difference between the studied groups as regards liver function tests. Also, high significant difference was recorded regarding CCR6 RNA expression, CTCs count and hepatic focal lesion detection by ultra sound. No significant difference between both groups regarding kidney function tests. Correlation studies between CTCs and other variables showed positive correlations with liver function tests (ALT, AST, BIL, Alb, AFP, and INR) and negative correlations with albumin and platelets.

Conclusion: CCR6 mRNA concentration was significantly increased in HCC patients and its elevation was correlated with CTCs percentage. Therefore, it can be concluded that the combined assessment of CTCs and CCR6 could be considered as noninvasive biomarkers for HCC patients.

Keywords: Hepatocellular carcinoma, Circulating tumor cells, Chemokine receptor 6, PCR, Flowcytomerter.

\section{INTRODUCTION}

Hepatocellular carcinoma (HCC) is the most common primary malignancy of the liver and is a leading cause of cancer-related death worldwide. In Egypt, it is the leading cause of cancer after hepatitis $\mathrm{C}$ infection, with male-to-female sex-linked infection $\{2: 1\}^{(1)}$.

Circulating tumor cells (CTCs) are cancer cells derived from the original tumor or metastatic foci that are flowing freely in the blood circulation. These calls are considered the drivers of recurrence and metastasis following liver cancer surgery for primary HCC. Those CTCs can lead to a new fatal metastasis and can be vividly described as "seeds" of tumors ${ }^{(2)}$. CTCs-positive rate was directly correlated with tumor size and counts as a biomarker of poor prognosis. The absolute numbers of CTCs detected have been associated with survival and treatment response and associated with increased recurrence risk after resection and shorter overall survival as the more advanced the cancer stage, the higher number of these cells in the peripheral blood ${ }^{(3)}$.

Hepatocellular carcinomas can synthesize various tumor-related proteins, polypeptides, and isoenzymes as well as the corresponding mRNAs. It is important to have tumor-specific markers of HCC to detect CTCs in the blood stream or lymphatic system like some cytokines (IL8 and TGF- $\beta 1$ ), AFP, alpha albumin, heat shock protein and group of chemokine receptors, which represent a very promising group of markers to detect CTC such as CXCR4, CX3CR1, and CCR6 express during HCC progression ${ }^{(4)}$.

Many studies have been conducted on chemokines and their receptors (chemokines-receptors axes) in HCC. Currently, the CCL20-CCR6 axis have received much research attention. CCL19, CCL20, CCL21, CXCL12 and the expression of their receptors were also studied in HCC, and found that all chemokines are expressed in normal liver and HCC tissues. Yet, CCL20 was the only chemokine, which showed significant upregulation along with its receptor CCR6 in HCC tissues ${ }^{(5)}$. The CCL20-CCR6 axis plays an important role in the growth and progression of HCC, as there is a strong association between the levels of their expression and the degrees of differentiation of $\mathrm{HCC}$, that is, with high differentiation, the expression was low and so there is a distinct increase in CCL20 and CCR6 expression rates in HCC tissues of grade III tumors in comparison to grade $\mathrm{II}^{(\mathbf{6})}$.

Screening methods for HCC include radiological tests such as ultra-sonography, computerized tomography, and magnetic resonance imaging. Serological methods such as liver function tests (ALT, AST, Bilirubin, INR, Albumin, and AFP). Percutaneous liver biopsy is yet used as diagnostic method for HCC, but its use is quite limited as being an invasive technique. Limitation of imaging and 
pathological examinations affects diagnostic accuracy and sensitivity, meanwhile common serum markers display poor diagnostic performance. Therefore, searching for new diagnostic methods becomes a critical issue. The availability of an effective method for the identification of CTCs in the blood and the investigation of their biological characteristics may promote the early diagnosis of liver cancer and prediction of early metastasis ${ }^{(7)}$.

The aim of this study was to assess the feasibility of using CTCs and CCR6 as non-invasive biomarkers for patients with $\mathrm{HCC}$.

\section{PATIENTS AND METHODS}

\section{Study design and population:}

This was a case control study conducted on 71 Egyptian HCC patients diagnosed by liver biopsy, CT scan, or MRI and 20 age and gender matched healthy subjects as control group. All subjects were recruited from the Outpatient Clinics and Inpatient of internal medicine Department, Ain Shams University Hospitals. The laboratory investigations were performed at Ain Shams University Hospital's Central Lab. The enumeration of CTCs and CCR6 mRNA expression were done at the Genetic Engineering and Biotechnology Research Institute, University of Sadat. All patients were subjected to detailed medical history and clinical examination. Laboratory investigations were performed for all participants.

\section{Sample collection and preparation:}

Blood samples were collected by venipuncture into a set of Vacutainer tubes for routine lab work and separation of the Peripheral Blood Mononuclear cells "PBMCs" that were used in RT-PCR and Flow Cytometry analyses. PBMCs were separated using ficoll-paque plus density (1077 g / L) using "Amersham biosciences Kit" according to the manufacturer's instructions.

\section{Routine laboratory investigations:}

Complete blood count (CBC) performed by 5 part differential automated cell counter Beckman Coulter $^{\circledR}$ LH 750 (Coulter Corporation, Florida, USA), prothrombin time (PT, INR) done on fully automated blood coagulation analyzer STA Compact Max-Stago (Asnieres Sur Seine Cedex, France).

Serum ALT, AST, total bilirubin, direct bilirubin, albumin, Alpha fetoprotein (AFP), and serum creatinine were performed on Beckman coulter AU 480 system (Beckman coulter, Inc. 250s. Kraemer Blvd. Brea, CA92821, USA).

\section{Estimation of chemokine receptor 6 (CCR6) by real time PCR:}

Genomic RNA extraction was done using "Pure link RNA mini kit "supplied by "Ambion" (Life technologies, Carlsbad, USA) according to the manufacturer's instructions. The collected RNA eluted was aliquoted into sterile tubes and stored at $-80^{\circ} \mathrm{C}$ until further processing. The cDNA was synthesized from the extracted RNA by using a high capacity cDNA reverse transcription kit supplied by "Applied Biosystems" (Life technologies, Carlsbad, USA) according to the manufacturer's instructions. Quantitative real-time PCR was done using a Light Cycler System (DT prime thermal cycler) (DNA Technology, Moscow, Russia) with CCR6-specific primers 5'-CCTAGCGGAGTTCCAGCAAA - $3^{\prime}$ (forward) and 5'-AATTCCAGCTGTCCCCTAGC-3' (reverse). Glyceraldehyde-3-phosphate dehydrogenase (GAPDH) was amplified as an internal control "Ambion" (Life technologies, Carlsbad, USA). The PCR mix contained $3.2 \mu \mathrm{L}$ Nuclease free $\mathrm{H} 2 \mathrm{O}$, $0.8 \mathrm{dNTP}$ mix, $2 \mu \mathrm{L}$ RT random primer, $1 \mu \mathrm{l}$ multiscribe reverse transcriptase, $2 \mu 1$ RT buffer and 1 $\mu \mathrm{L}$ RNase inhibitor. This PCR mix was pipetted into a 96 well reaction plate. The plate was placed into the rotor of the Light Cycler, and the samples were amplified. PCR cycles were monitored continuously with the maxima SYBR Green q PCR master mix supplied by "Thermofisher scientific" (Life technologies, Carlsbad, USA). The system software records the fluorescence measurements of internal control and CCR6 gene from each well made during the plate read, and then CCR6 RNA concentration was calculated from the following equation: CCR6 RNA concentration $=2^{\Delta \mathrm{ct}}(\Delta \mathrm{ct}=\mathrm{CCR} 6$ reading $-\mathrm{GAPDH}$ reading).

\section{Identification of CTCs by Flow Cytometry:}

Flow cytometric measurement of CTCs was performed using "BD Accuri C6 plus" flow cytometry (BD Life sciences Inc, USA). Anti-human anti-CD45 and anti-CK19 monoclonal antibody was used to identify CTCs as cells negative for CD45 and positive for CK19 $\left(\mathrm{CD} 45^{-} \mathrm{CK} 19^{+}\right)$in the separated mononuclear layer. The fluorescence of the delimited cells is analyzed to distinguish the positively stained cells from the negative unstained ones. The results were then expressed as numbers of $\mathrm{CD} 45^{-} \mathrm{CK} 19^{+}$ CTCs in relation to all cells acquired by the cytometer.

\section{Ethical Approval:}

The study protocol gained approval from the Local Ethics' Committee of the Internal Medicine Department, Ain Shams University Hospital. The nature of the study was explained and an informed consent was obtained from all participants before enrollment in the study.

\section{Statistical analysis}

Results were analyzed using SPSS version 24. Quantitative data were expressed as mean \pm standard deviation (SD). Qualitative data were expressed as frequency and percentage. Chi-square test was used to 
compare qualitative variables. Independent t-test was used to compare two independent quantitative variables. ANOVA test was used to compare more than two groups.

Spearman's correlation co-efficient test was used to assess the relationship between variables in the same group. Receiver-operating characteristic (ROC) curve analysis was used to examine the value of CTC and CCR6 for discrimination between cases and controls. A p value $\leq 0.05$ was considered statistically significant.

\section{RESULTS}

\section{Clinical characteristics:}

Ninety-one subjects that enrolled in the study were categorized into 2 groups; (patient group) 71

Table (1): Clinical characteristics and laboratory findings of the studied groups

\begin{tabular}{|c|c|c|c|c|}
\hline \multicolumn{2}{|c|}{ Groups Variables } & $\begin{array}{l}\text { Control Group } \\
\quad(\mathbf{n}=\mathbf{2 0})\end{array}$ & $\begin{array}{c}\text { Patient Group } \\
\quad(n=71)\end{array}$ & P-value \\
\hline \multirow{3}{*}{$\begin{array}{l}\text { Child classification } \\
\%\end{array}$} & Negative & $20(100.0 \%)$ & $0(0.00 \%)$ & \multirow{3}{*}{0.0001} \\
\hline & $\mathbf{A}_{5}$ & $0(0.00 \%)$ & $58(81.7 \%)$ & \\
\hline & $\mathbf{A}_{6}$ & $0(0.00 \%)$ & $13(18.3 \% 0$ & \\
\hline \multirow{2}{*}{$\begin{array}{l}\mathrm{HCV} \\
\%\end{array}$} & Negative & $20(100.0 \%)$ & $8(11.3 \%)$ & \multirow{2}{*}{0.0001} \\
\hline & Positive & $0(0.00 \%)$ & $63(88.7 \%)$ & \\
\hline \multirow{2}{*}{$\begin{array}{l}\text { HbsAg } \\
(\%)\end{array}$} & Negative & $20(100.0 \%)$ & $63(88.7 \%)$ & \multirow{2}{*}{0.125} \\
\hline & Positive & $0(0.00 \%)$ & $8(11.3 \%)$ & \\
\hline \multirow{2}{*}{$\begin{array}{l}\text { Ascites } \\
(\%)\end{array}$} & No & $20(100.0 \%)$ & $40(56.3 \%)$ & \multirow{2}{*}{0.0001} \\
\hline & Yes & $0(0.00 \%)$ & $(43.7 \%) 31$ & \\
\hline \multicolumn{2}{|c|}{ Focal lesion by U/S Mean \pm SD } & $0.00 \pm 0.00$ & $8.49 \pm 10.09$ & 0.0001 \\
\hline \multicolumn{2}{|l|}{ ALT (U/L) } & $28.05 \pm 5.93$ & $40.39 \pm 2.79$ & 0.0001 \\
\hline \multicolumn{2}{|l|}{$\operatorname{AST}(\mathbf{U} / \mathbf{L})$} & $27.10 \pm 5.49$ & $46.27 \pm 4.57$ & 0.0001 \\
\hline \multicolumn{2}{|l|}{ T.Bili (mg/dl) } & $0.99 \pm 0.09$ & $1.22 \pm 0.43$ & 0.0001 \\
\hline \multicolumn{2}{|l|}{ D.Bili (mg/dl) } & $0.41 \pm 0.16$ & $0.56 \pm 0.15$ & 0.006 \\
\hline \multicolumn{2}{|l|}{ Alb (g/dL) } & $4.05 \pm 0.34$ & $3.47 \pm 0.58$ & 0.0001 \\
\hline \multicolumn{2}{|l|}{ INR } & $1.08 \pm 0.089$ & $1.24 \pm 0.27$ & 0.0001 \\
\hline \multicolumn{2}{|l|}{$\mathrm{AFP}(\mathrm{ng} / \mathrm{ml})$} & $1.75 \pm 0.48$ & $117.90 \pm 21.24$ & 0.0001 \\
\hline \multicolumn{2}{|l|}{ Creatinin(mg/dl) } & $0.99 \pm 0.16$ & $1.02 \pm 0.19$ & 0.507 \\
\hline \multicolumn{2}{|c|}{ Hemoglobin (Hb) (g/dl) } & $13.08 \pm 0.58$ & $12.32 \pm 1.38$ & 0.001 \\
\hline \multicolumn{2}{|l|}{ TLC(x 10 $13 /$ ul $)$} & $4.84 \pm 1.05$ & $6.32 \pm 1.56$ & 0.0001 \\
\hline \multicolumn{2}{|l|}{ Platelet(x 10 $3 /$ ul) } & $273.95 \pm 48.79$ & $181.21 \pm 8.84$ & 0.0001 \\
\hline \multicolumn{2}{|c|}{ CCR6 RNA concentration } & $0.19 \pm 0.16$ & $11.74 \pm 1.75$ & 0.0001 \\
\hline \multicolumn{2}{|l|}{ CTCs \% } & $0.00 \pm 0.00$ & $13.93 \pm 3.32$ & 0.0001 \\
\hline
\end{tabular}

\section{CTCs enumeration by flow cytometry:}

CTCs enumeration was done using flow cytometry as CD45- CK19+ cells (Figure 1), and it was found significantly higher in HCC patients compared to the controls $(\mathrm{P} \leq 0.0001)$.
HCC patients $[6(8.5 \%)$ males and $65(91.5 \%)$ females].

The patients' ages ranged from 50- 61 years old with mean of $56.20 \pm 5.53$ years. Twenty age- and gender-matched healthy subjects were recruited as control group, [0 (0\%) male and 20 (100\%) female]. Their ages ranging from 31- 42 years with mean of $37.00 \pm 5.22$ years.

Table (1) showed clinical characteristics and laboratory findings of the studied groups. A comparative study by Chi-square test $(\chi 2)$ showed statistically significant difference in all studied parameters except for $\mathrm{HbsAg}$ and serum creatinine. 

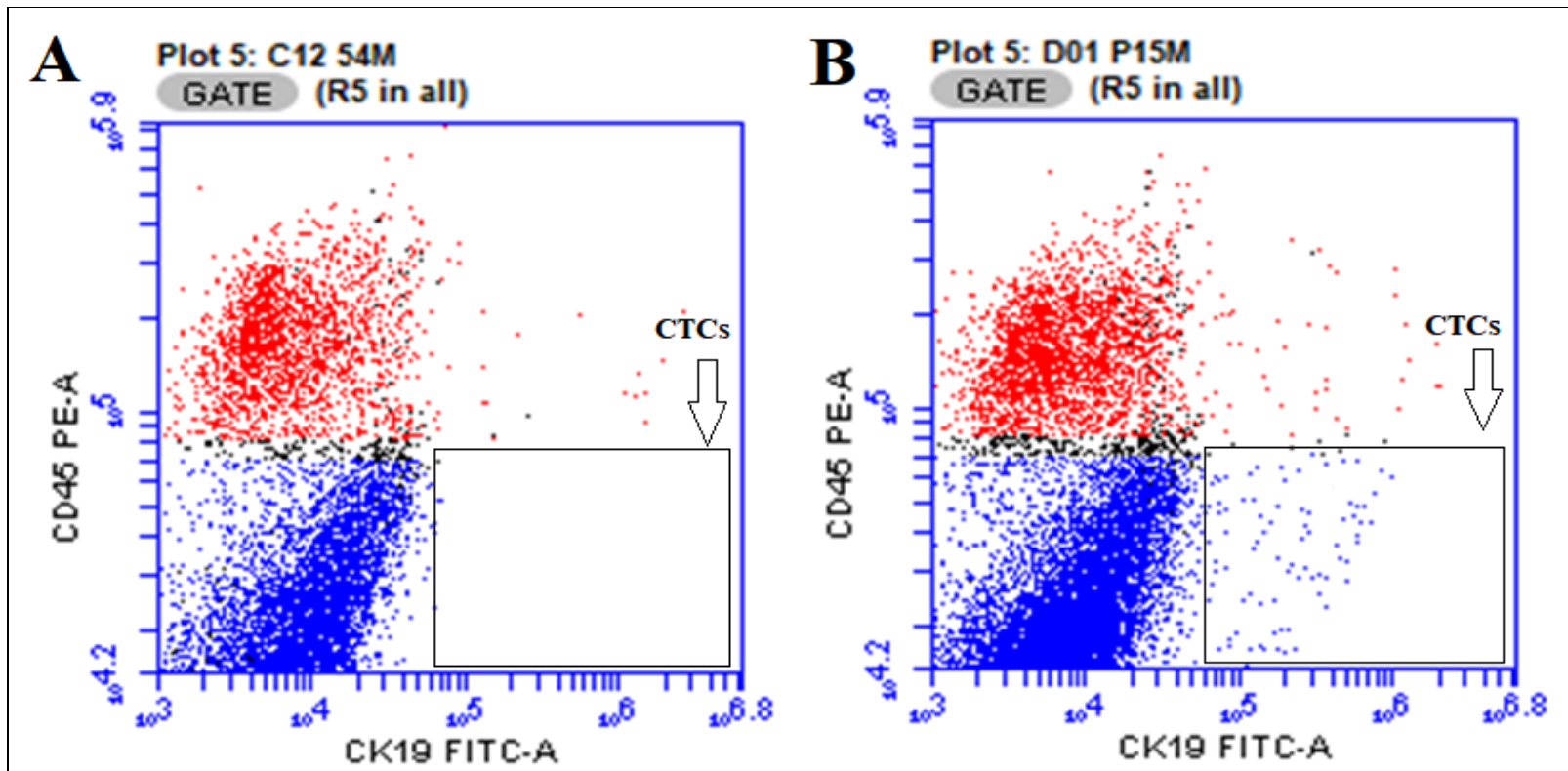

Figure (1): Flow cytometry detected small numbers of (0\%-0.05\%) of CD45 CK19+ cells in the blood of (A) a healthy control, and (B) HCC patient. The dot plots represent 1 sample in each group, respectively.

\section{Real time PCR of CCR6 mRNA:}

CCR6 expression was found significantly higher in the HCC patients compared to controls $(\mathrm{P}<0.0001)$ (Table 1).

\section{Diagnostic performance of CCR6 and CTC in discrimination of patient and control groups:}

Using ROC curve, it was shown that CCR6 at the cutoff $>1.02$ can be used to discriminate between patients and controls with $93.1 \%$ sensitivity, $78.8 \%$ specificity, $88.5 \%$ PPV and $86.7 \%$ NPV, while CTC concentration $>3.5$ was the cutoff between patients and controls with $91.4 \%$ sensitivity, $81.8 \%$ specificity, $89.8 \%$ PPV and $84.4 \%$ NPV (Figure 2).

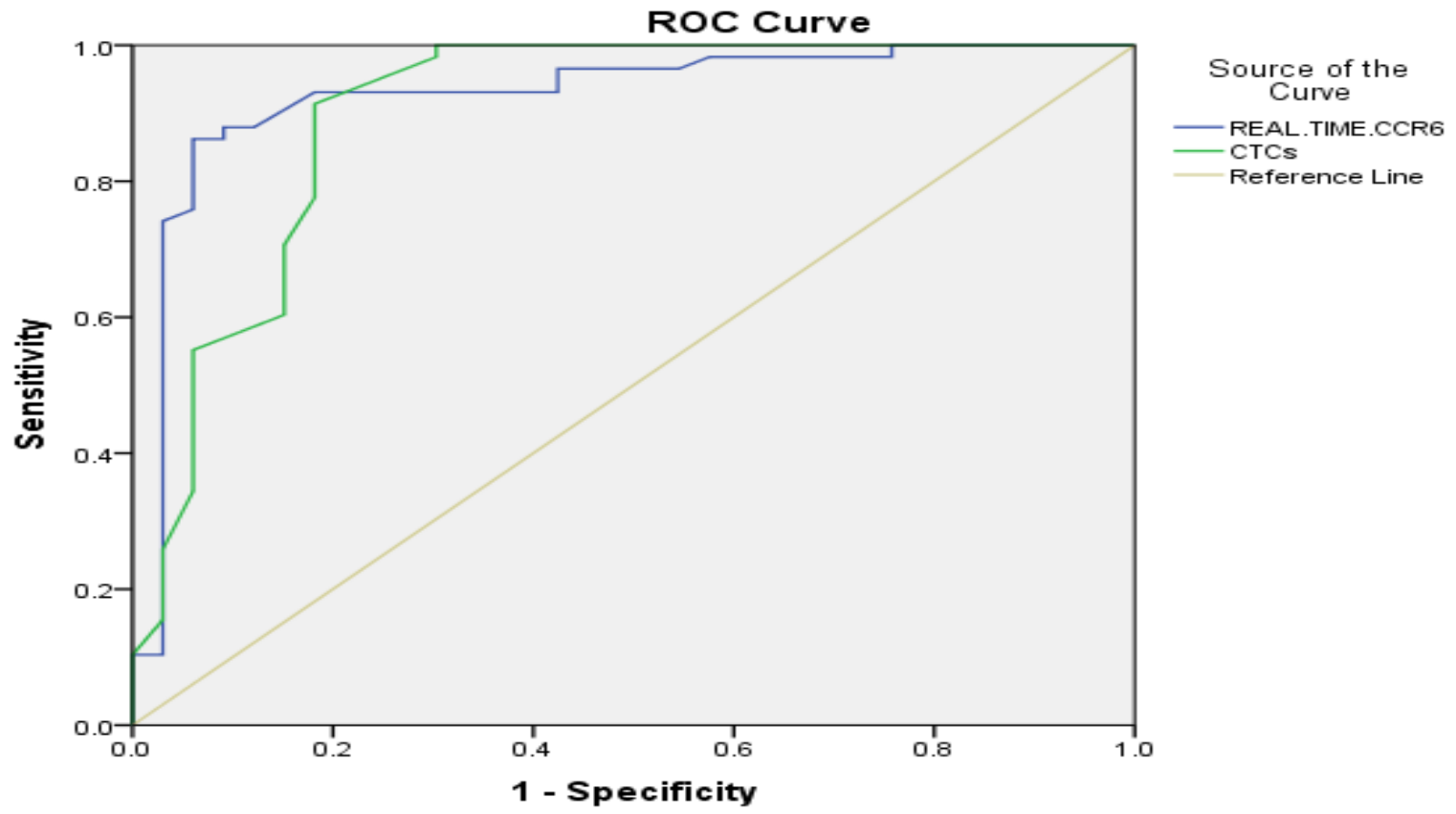

Figure (2): Receiver operating characteristic (ROC) curve between patient and control group as regards CCR6 and CTCs.

\begin{tabular}{|l|c|c|c|c|c|c|c|}
\hline & Cut off & AUC & Sensitivity & specificity & PPV & NPV & P-value \\
\hline CCR6 & $>1.020$ & 0.927 & 93.1 & 78.8 & 88.5 & 86.7 & 0.01 \\
\hline CTCs & $>5.30$ & 0.900 & 91.4 & 81.8 & 89.8 & 84.4 & 0.01 \\
\hline
\end{tabular}

AUC: area under the curve, PPV: positive predictive value, NPV: negative predictive value 


\section{Clinical correlations:}

There was a significant positive correlation between CTCs and Real time CCR6 (p value <0.05) (Figure 3), but no significant correlation was found between CTCs and the number of Focal lesions ( $\mathrm{p}$ value $>0.05$ ).

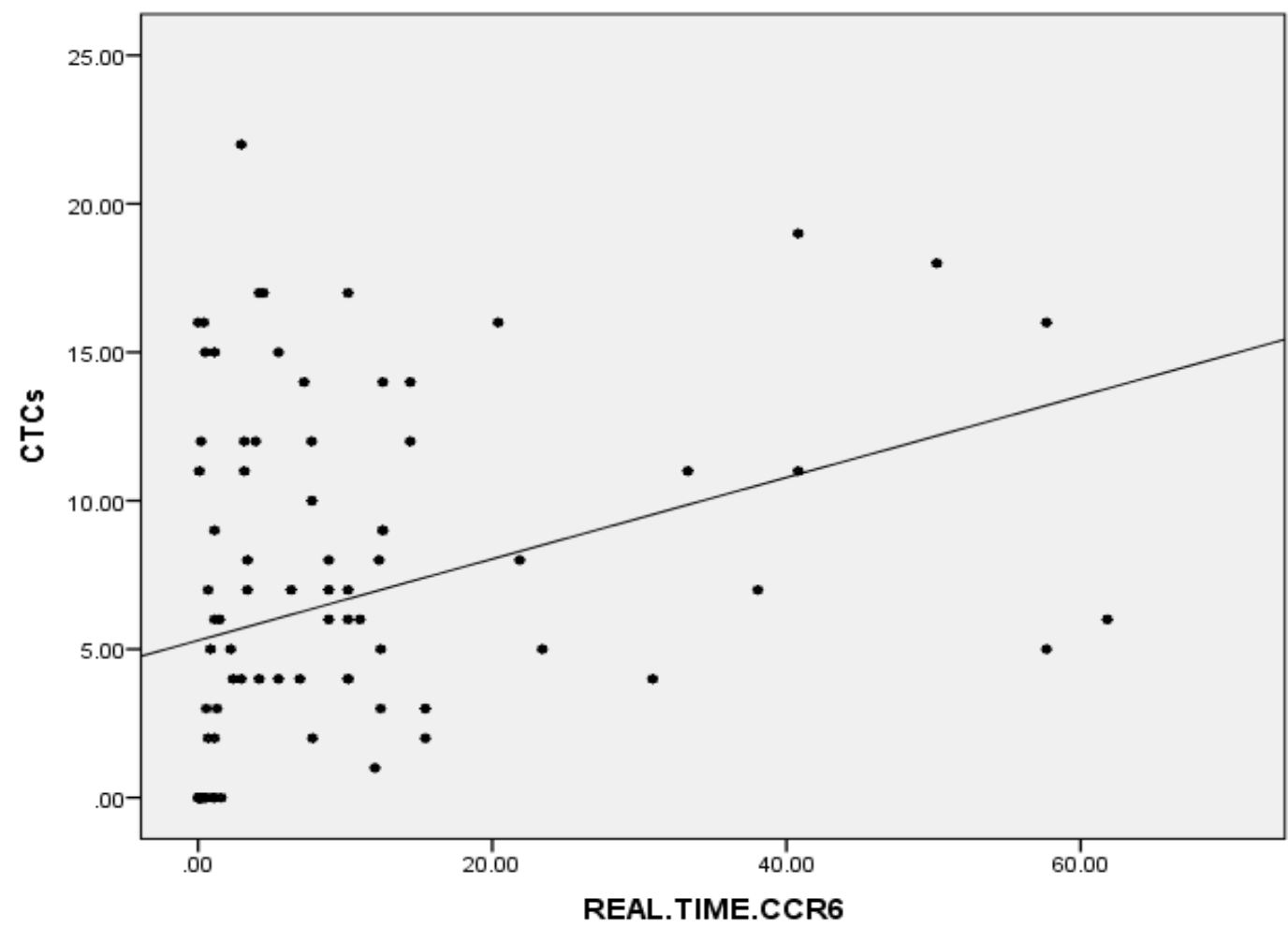

Figure (3): Correlation between the CTCs and CCR6 expression levels.

There was significant positive correlation between CTCs and ALT, INR, AFP and creatinine where the $r$ values were equal to $0.292,0.274,0.431$ and 0.226 respectively ( $\mathrm{p}$ value $<0.05$ ). While, there was significant negative correlation between CTCs and Alb and Platelet, with $r$ values of -0.277 and -0.361 respectively ( $p$ value $<0.05$ ). There was no significant correlation found between CTCs and AST, T.Bili, D.Bili, Hb and TLC. There was no significant correlation between CCR6 and all studied variables with the HCC group (p > 0.05) (Table 2).

Table (2): Correlation between the CTCs and different variables in case group

\begin{tabular}{|c|c|c|c|c|}
\hline \multirow{2}{*}{ Variables } & \multicolumn{2}{|c|}{ CTCs $(n=71)$} & \multicolumn{2}{|c|}{$\operatorname{CCR6}(n=71)$} \\
\hline & $\mathrm{R}$ & $\mathrm{P}$ & $\mathrm{R}$ & $\mathrm{P}$ \\
\hline ALT (U/L) & 0.292 & $<0.05$ & 0.199 & $>0.05$ \\
\hline AST (U/L) & 0.044 & $>0.05$ & 0.081 & $>0.05$ \\
\hline T.Bili (mg/l) & 0.075 & $>0.05$ & 0.062 & $>0.05$ \\
\hline D.Bili (mg/dl) & 0.121 & $>0.05$ & 0.079 & $>0.05$ \\
\hline Alb (g/dl) & -0.277 & $<0.05$ & 0.064 & $>0.05$ \\
\hline INR & 0.274 & $<0.05$ & 0.051 & $>0.05$ \\
\hline AFP (ug/l) & 0.431 & $<0.05$ & 0.035 & $>0.05$ \\
\hline Creat. (g/dl) & 0.226 & $<0.05$ & 0.068 & $>0.05$ \\
\hline Hb (g/dl) & -0.023 & $>0.05$ & 0.028 & $>0.05$ \\
\hline TLC & 0.062 & $>0.05$ & 0.083 & $>0.05$ \\
\hline Platelet & -0.361 & $<0.05$ & 0.005 & $>0.05$ \\
\hline
\end{tabular}




\section{DISCUSSION}

$\mathrm{HCC}$ is the fourth common cancer in Egypt and the sixth most common cancer worldwide. In 2010, HCC was responsible for approximately $9.1 \%$ of all deaths (746,000 deaths), and in Egypt, HCC is the most common cause of cancer-related death and morbidity ${ }^{(8,9)}$.

There is an increasing interest about the role of CCR6-CCL20 axis in HCC as the only chemokinechemokine receptor showing significant up-regulation in the carcinoma tissues. Moreover, there is strong association between their level of expression and the degree of cancer differentiation as the higher is the expression the lower is tumor differentiation, so their expression is higher in grade III HCC than grade II $^{(\mathbf{1 0})}$. In the present study, we aimed to identify the role of CTC and CCR6 in hepatocellular carcinoma patient. The study was conducted on 91 subjects; 71 HCC patients (patient group) and 20 age- and gendermatched healthy subjects (control group).

In this study, we measured CCR6 mRNA gene expression by real time PCR and the results showed more prominent gene expression in HCC patients compared to normal controls. This result is in accordance with the study carried out by Liu and Wu (11) who reported that HCC patients showed elevated levels of CCR6 and that it could be used to differentiate HCC from those with no detected focal lesion. Rubie et al. ${ }^{\left({ }^{(6)}\right.}$ stated that CCR6 at cut-off 1.0 could not discriminate normal subjects from hepatoma patients especially those in the early stages of the disease with low CCR6 expression over tumor cells those with any other GIT tumors as colon cancer.

Abnormal high expression of CCR6/CCL20 participates and may be an important factor for the occurrence and development of liver cancer, as Hu et al. ${ }^{(12)}$ found that an increase in the expression of CCR6/CCL20 is associated with the malignant biological behavior of HCC. Support for this theory comes from the greater tendency of the occurrence of vascular invasion, intrahepatic metastasis, pulmonary metastasis and other malignant activities in CCR6positive HCC patients, after adjustment for the clinical influence factors of gender, age, tumor size and degree of differentiation ${ }^{(13,14)}$. In contrast to this, Rubie et al. (6) stated that serum levels of CCR6 is considered a less reliable marker of HCC as its elevated level may be related to other GIT tumors as cancer colon. In addition, Ghadjar et al. ${ }^{(15)}$ emphasized that CCR6 is not a hepatoma specific biomarker, since elevations were reported in other cancer as colorectal cancers and colorectal liver metastases.

Using Roc curve analysis, the results revealed that cut-off value $>1.02$ for CCR6 mRNA concentration could discriminate patients from controls with $93.1 \%$ sensitivity, $78.8 \%$ specificity, $88.5 \%$ positive predictive value (PPV) and $86.7 \%$ negative predictive value (NPV). While cutoff values for CTC numbers > 3.5 could differentiate between patients and controls with $91.4 \%$ sensitivity, $81.8 \%$ specificity, and $89.8 \% \mathrm{PPV}$ and $84.4 \% \mathrm{NPV}$.

Liquid biopsies, specifically for CTCs detection, can make up for the deficiencies of tissue biopsy. CTCs specimens are obtained from peripheral blood and can reflect the current state of the disease, we identified CTCs by flow cytometry through detection of a surface marker on it (CD45 CK19 ${ }^{+}$ cells), and the results showed significant increased expression of CK19 in HCC patients compared to controls. Statistical analysis of this finding and the correlation with focal lesion detected by ultrasound increases the value of CTCs as a noninvasive marker for HCC and this agrees with Shibuta et al. ${ }^{(16)}$ who reported that elevated levels of CTCs were detected in HCC patients, and that is positively correlated with numbers of focal lesions in the liver. In the present study, mRNA CCR6 did not correlate with any of the clinical or biochemical parameters studied. However, CTCs were found correlated positively with serum concentrations of ALT, INR, AFP and creatinine.

AST and ALT are excellent markers of hepatocellular injury. They participate in gluconeogenesis by catalysing the transfer of amino groups from aspartic acid or alanine to ketoglutaric acid to produce oxaloacetic acid and pyruvic acid respectively. In this study we found a significant increase in liver function tests (ALT, AST and total bilirubin) in HCC patients compared to controls. This agrees with Balkwill and Mantovani ${ }^{(17)}$ who reported significant elevated serum level of ALT, AST and total bilirubin in patients with HCC.

We detected an increase in INR levels in HCC patients group compared to control group. Zhang et al. ${ }^{(18)}$ had the same finding in their study and reported that INR level was increased in HCC patients, especially with DM, and these patients should be monitored for coagulation function in clinical practice. Schimanski et al. ${ }^{(19)}$ also found that elevated INR levels are strongly associated with HCC, up to three times the normal level in some patients.

AFP remains the most used biomarker during the management of HCC. AFP is a glycoprotein that is elevated in the serum of approximately $70 \%$ of patients with HCC. In the current study, serum AFP was significantly higher in the patient group compared to control group $(\mathrm{p}<0.0001)$. This agrees with Schimanski et al. ${ }^{\left({ }^{(19)}\right.}$ who reported that elevated serum levels of AFP is a diagnostic marker for HCC as its serum levels always exceed twice its reference range. However, some other studies demonstrated that serum AFP value is a modest diagnostic marker for $\mathrm{HCC}$ due to its low specificity. Despite of that the prognostic role of AFP in HCC remains obscure ${ }^{(20)}$. Given the fact that CTCs is a subpopulation of cells that express an 
epithelial-to-mesenchymal phenotype during which cancer cells disseminate from the tumor to distant metastatic sites ${ }^{(\mathbf{2 1})}$. Therefore, CTCs could be considered a prognostic marker for patient outcome in advanced stages of HCC. Our findings that CTCs correlate positively with serum AFP may support the prognostic value of AFP.

\section{CONCLUSION}

CTCs numbers are increased in patients with HCC and they are found positively correlated with serum ALT, creatinine, INR, and AFP. CCR6 mRNA levels were also significantly increased in HCC patients and they were correlated with CTCs numbers. Thus, it can be concluded that the combined assessment of CTCs and CCR6 may be used as noninvasive biomarkers for HCC patients.

Funding: This research did not receive any specific grants from funding agencies in the public, commercial, or non-profit sectors.

\section{Conflict of interest: None.}

\section{REFERENCES}

1. Nathan H, Pawlik T (2011): Staging of hepatocellular carcinoma. In: Masters KM, Vauthey J-N, editors. Hepatocellular Carcinoma. Berlin, Germany: Springer, Pp: 69-80.

2. Jin T, Peng H, Wu H (2013): Clinical value of circulating liver cancer cells for the diagnosis of hepatocellular carcinoma: A meta-analysis. Biomedical Reports, 1: 731-736.

3. Alix-Panabières C, Pantel K (2016): Clinical applications of circulating tumor cells and circulating tumor DNA as liquid biopsy. Cancer Discov, 6 (5): 479-91.

4. Chiappini F (2012): Circulating Tumor Cells Measurements in Hepatocellular Carcinoma. International Journal of Hepatology. https://DOI:10.1155/2012/684802.

5. Fujii H, Itoh Y, Yamaguchi K et al. (2004): Chemokine CCL20 enhances the growth of HuH7 cells via phosphorylation of p44/42 MAPK in vitro. Biochem Biophys Res Commun., 322: 1052-1058.

6. Rubie C, Frick V, Wagner M et al. (2006): Enhanced expression and clinical significance of CC-chemokine MIP-3 alpha in hepatocellular carcinoma. Scand J Immunol., 63: 468-477.

7. Wang C, Wang X, Gong G et al. (2012): Increased risk of hepatocellular carcinoma in patients with diabetes mellitus: a systematic review and meta- analysis of cohort studies. Int J Cancer, 130 (7): 1639 1648.

8. Abd-Elsalam S, Elwan N, Soliman H et al. (2018): Epidemiology of liver cancer in Nile delta over a decade: a single-center study. South Asian J Cancer, 7 (1): 24-26.

9. Villanueva A (2019): Hepatocellular Carcinoma. N Engl J Med., 380 (15): 1450-1462.

10. Schutyser E, Struyf S, Van Damme J (2003): The CC chemokine CCL20 and its receptor CCR6. Cytokine \& Growth Factor Rev., 14 (5): 409-426.

11. Liu Y, Wu F (2010): Global burden of aflatoxininduced hepatocellular carcinoma: a risk assessment. Environ Health Perspect., 118 (6): 818824.

12. Hu D, Du C, Xue W et al. (2013): The expression of chemokine receptors CCR6, CXCR2 and CXCR4 is not organ-specific for distant metastasis in colorectal cancer: A comparative study. Histopathology, 63: 167173.

13. Uchida H, Iwashita Y, Sasaki A (2006): Chemokine receptor CCR6 as a prognostic factor after hepatic resection for hepatocellular carcinoma. J. Gastroenterol. Hepatol., 21: 161-168.

14. Du D, Liu Y, Qian H et al. (2014): The effects of the CCR6/CCL20 biological axis on the invasion and metastasis of hepatocellular carcinoma. Int J Mol Sci., 15 (4): 6441-6452.

15. Ghadjar P, Coupland S, Na I et al. (2006): Chemokine receptor CCR6 expression level and liver metastasis in colorectal cancer. J Clin Oncol., 24: 1910 16.

16. Shibuta K, Mori M, Shimoda $K$ et al. (2002): Regional expression of CCL20/CCR6 in liver and hepatocellular carcinoma and cell-cycle variation during in vitro differentiation. Jpn J Cancer Res., 93: 789-797.

17. Balkwill F, Mantovani A (2016): Inflammation and cancer: back to Virchow? Lancet, 357: 539-545.

18. Zhang H, Gao C, Fang L et al. (2013): Increased International normalized ratio level in hepatocellular carcinoma patients with diabetes mellitus. World J Gastroenterol., 19 (15): 2395-2403.

19. Schimanski C, Bahre R, Gockel I et al. (2017): Dissemination of hepatocellular carcinoma is mediated via chemokine receptor CCR6. Br J Cancer, 95: 210217.

20. Park H, Park J (2013): Clinical significance of AFP and PIVKA-II responses for monitoring treatment outcomes and predicting prognosis in patients with hepatocellular carcinoma. Biomed Res Int., 2013: 310427.

21. Giannelli G, Koudelkova P, Dituri F et al. (2016): Role of epithelial to mesenchymal transition in hepatocellular carcinoma. Journal of Hepatology, 65 (4): 798-808. 